\title{
"The More They Know, the Better Care They Can Give": Patient Perspectives on Measuring Functional Status in Primary Care
}

\author{
Francesca M. Nicosia, $P h D^{1,2}$ (D), Malena J. Spar, MPH ${ }^{1,2}$, Alicia Neumann, $P h D^{2}$, \\ Molly C. Silvestrini, $B A^{1,2}$, Maureen Barrientos, $B A^{7,2}$, and Rebecca T. Brown, MD, MPH ${ }^{3,4,5}$
}

\begin{abstract}
'San Francisco VA Health Care System, San Francisco, CA , USA; '2Division of Geriatrics, Department of Medicine of the University of California, San Francisco, San Francisco, CA , USA; ${ }^{3}$ Geriatrics and Extended Care, Corporal Michael J. Crescenz VA Medical Center , Philadelphia , PA , USA; ${ }^{4}$ Center for Health Equity and Research Promotion, Corporal Michael J. Crescenz VA Medical Center, Philadelphia , PA , USA; ${ }^{5}$ Division of Geriatric Medicine, Perelman School of Medicine of the University of Pennsylvania , Philadelphia , PA , USA.
\end{abstract}

\begin{abstract}
BACKGROUND: Despite its importance to care and outcomes for older adults, functional status is seldom routinely measured in primary care. Understanding patient perspectives is necessary to develop effective, patientcentered approaches for measuring function, yet we know little about patient views on this topic.
\end{abstract}

OBJECTIVE: To examine patient and caregiver perspectives on measuring activities of daily living (ADLs) and instrumental ADLs (IADLs).

DESIGN: Qualitative study using semi-structured interviews.

PARTICIPANTS: Twenty-eight patients aged 65 or older and five caregivers in primary care clinics at one Veterans Affairs Medical Center.

APPROACH: We conducted interviews to elicit patient and caregiver perspectives on the importance of measuring function, including preferences for method of screening and assessment, wording of questions, and provider communication style. We analyzed interviews using qualitative thematic analysis.

KEY RESULTS: We identified several themes related to measuring function in primary care. First, most participants reported that measuring function is part of quality, holistic care. However, a minority of participants noted that discussing function, especially IADLs, was not medically relevant. Second, in terms of preferences for measuring function, participants noted that interdisciplinary approaches to measuring function are optimal and that face-to-face assessment is most "intimate" and can prompt reflection on one's limitations. However, some participants indicated that self-assessment is less invasive than inperson assessment. Third, participants had varied preferences regarding communicating about function. Participants noted that asking about difficulty with activities versus need for help are distinct and complementary concepts and that providing context is essential when discussing sensitive topics such as functional decline.

Erancesca M. Nicosia and Malena J. Spar contributed equallu to this work.

Prior Presentation This manuscript was presented as a poster at the 2019 Annual Scientific Meeting of the American Geriatrics Society in Portland OR on Mau 2.2019.

Received August 1, 2019

Accepted July 17, 2020

Published online August 4, 2020
CONCLUSIONS: Most patients and caregivers reported that measuring function was important, preferred faceto-face assessment, and emphasized the importance of providing context when asking about function. These findings suggest that incorporating patient and caregiver preferences for measuring function can improve satisfaction and experience with functional assessment in primary care.

KEY WORDS: functional status; functional assessment; primary care; older adults; qualitative research.

J Gen Intern Med 35(10):2947-54

DOI: $10.1007 / \mathrm{s} 11606-020-06075-8$

(c) Society of General Internal Medicine (This is a U.S. government work and not under copyright protection in the U.S.; foreign copyright protection may apply) 2020

\section{INTRODUCTION}

Older adults' ability to perform activities of daily living (ADLs), such as bathing and dressing, is central to their quality of life and health. When older adults develop difficulty performing ADLs, often called functional impairment, they are at higher risk for adverse outcomes including hospitalization, nursing home admission, and death. ${ }^{1-3}$ Maintaining function is one of the health outcomes that matters most to older adults, ${ }^{4}$ with losing independence a greater fear than financial hardship or death. ${ }^{5,6}$ Understanding function is also key to providing patient-centered care, including predicting how patients will tolerate interventions, ${ }^{7-11}$ individualizing cancer screening, ${ }^{12}$ and helping plan for long-term services and supports. Yet despite the central importance of function to older adults' quality of life and health, it is seldom routinely measured in primary care. ${ }^{13-16}$

Although relatively little is known about the reason for this disconnect, some studies have identified provider and system barriers to routine functional status measurement in primary care, including time pressures and poor integration of measurement into clinical workflows. ${ }^{17-19}$ These findings could help to inform provider-centric aspects of measurement, including improved workflows, more time efficient approaches 
to measurement, and streamlined referrals for services and supports. ${ }^{18}$ However, little is known about older adults' perspectives and preferences for functional status measurement. ${ }^{20}$ Understanding older adults' perspectives is key to develop patient-centered approaches to functional assessment that account for preferences for mode of screening and assessment, wording of questions, and provider communication style.

As part of a larger study focused on improving measurement of functional status in Veteran Affairs (VA) primary care clinics, we conducted a qualitative study among older veterans and caregivers to explore their perspectives on functional status measurement.

\section{METHODS}

\section{Design}

In this qualitative study, older veterans and caregivers participated in semi-structured interviews about their perspectives on measuring function in primary care. The institutional review boards of the San Francisco VA Medical Center, the Corporal Michael J. Crescenz VA Medical Center, and the University of California, San Francisco, approved the study.

\section{Setting and Participants}

We recruited participants from primary care clinics at one VA medical center. We first identified all patients ages 65 or older who had seen their primary care provider in the past year. We asked providers to review patient lists and categorize patients' function as follows: (1) does not need help from another person to perform basic ADLs (i.e., bathing, dressing, eating, walking across a room, transferring, toileting); (2) needs help with 1-2 ADLs; or (3) needs help with 3 or more ADLs.

To select a diverse sample of patients, we used stratified purposive sampling. ${ }^{21}$ We stratified recruitment by funcitonal status, race/ethnicity, and gender. We mailed patients a letter explaining the study with a toll-free opt-out telephone number and a consent form. If patients did not opt-out within 7 days of the mailing date, a research assistant followed up to determine interest in participating and assess ability to provide informed consent using a teach-back method. ${ }^{22}$ Among those able to provide consent, we scheduled in-person interviews at the patient's home or the VA medical center. For patients with cognitive impairment who were unable to provide informed consent, we recruited their family caregiver using a similar process.

\section{Data Collection}

The study team consisted of a medical anthropologist (FN), a sociologist (AN), three clinical research assistants trained in qualitative methods (MB, MJS, MCS), and a geriatrician-researcher (RB). Between March 2016 and October 2016, FN conducted in-person interviews with each participant lasting approximately $60 \mathrm{~min}$.
Participants gave written consent before each interview. Interviews were audio recorded and professionally transcribed.

Using a semi-structured interview guide, we first asked an open-ended question about the patients' daily routine. Next, to assess ability to perform ADLs and IADLs, participants completed a card sort activity. ${ }^{23,24}$ The cards included graphics and text describing 8 ADLs (bathing, dressing, eating, grooming, using the toilet, transferring from a chair, transferring from bed, walking across a room), and 8 IADLs (shopping for groceries, preparing a hot meal, managing money, taking medication, doing housework, making phone calls, doing housework, driving, or using public transportation). We asked participants to categorize each activity as "easy," "somewhat difficult," or "difficult." We asked participants why they categorized the cards as they did and whether they received help with each activity. Next, participants categorized cards according to the importance of discussing each activity with their provider (very, somewhat, or not important). Participants reported why individual activities were or were not important to discuss with providers and any experiences discussing these activities in primary care.

To assess patients' preferences for method of functional status measurement and communication with providers, we presented clinical vignettes. ${ }^{25}$ We read a brief narrative describing three scenarios in which function was measured: (1) screening by a nurse, (2) assessment by a provider, and (3) patient self-assessment using paper or an electronic tablet or kiosk. We pilot tested the interview guide and card sort activity with the first five participants and revised the guide to reflect emergent constructs and themes.

\section{Data Analysis}

We used an inductive approach to qualitative thematic analysis to analyze transcripts. ${ }^{26,27}$ First, two members of the study team (FN, AN) independently reviewed five transcripts and developed codes to identify key concepts. Three team members (FN, AN, RB) then met to refine codes and draft the initial coding scheme. Next, five team members (FN, AN, MB, MJS, MCS) refined codes through serial review of transcripts and identified relationships between emerging themes. During this iterative process, we met to discuss findings after each set of three independently coded transcripts. We resolved disagreements about the presence, scope, or definition of codes and presence of themes through discussion and consensus. We aimed to identify themes representing a range of experiences within and across participant functional status groups. We conducted interviews until we had an adequate sample from which we observed no more emergent themes. ${ }^{28,}{ }^{29}$ We managed qualitative data using ATLAS.ti (Version 8, Berlin, Scientific Software Development). To protect confidentiality of two participants over 89 years of age, we altered the age of one individual \pm 2 years alongside a direct quotation in the "RESULTS" section and supplemental table. ${ }^{30}$ 


\section{RESULTS}

\section{Participant Characteristics (Table 1)}

Twenty-eight patients and five caregivers completed interviews. Patients' mean age was 81 years (standard deviation, 10), $45 \%$ were female, $70 \%$ were White, $15 \%$ were Black, and 9\% were Latino/a. Twenty-one percent of patients had a high school education or less. Over half were independent in ADLs (58\%), 21\% needed help with 1-2 ADLs, and 21\% needed help with more than 3 ADLs.

\section{Importance of Measuring and Discussing Function in Primary Care}

We identified several themes related to the importance of measuring function: (1) measuring function is part of quality, holistic care; (2) measuring function is not medically relevant; and (3) providers are not equipped to help with functional decline (Table 2).

Measuring Function Is Part of Quality, Holistic Care. Among participants who reported that assessing function was important, the most common theme was that measuring function is part of quality, holistic care. As an 85-year-old man needing help with 1-2 ADLs said, "Well, [the provider is] asking because they want you to continue in a good way. And it's their duty to make sure that you're well taken care of." A 101-year-old functionally independent man explained that measuring function was part of a provider's responsibility "to see how you're doing, [because] they should look after you."

Participants also noted that discussing function with providers is central to providing optimal care because it allows providers to proactively intervene to prevent functional

Table 1 Participant Characteristics

\begin{tabular}{ll}
\hline \hline Characteristics & Participants $(\boldsymbol{n = 3 3 )}$ \\
\hline Age, mean years (standard deviation) & $81( \pm 10)$ \\
Female, $n(\%)$ & $15(45 \%)$ \\
Functional status (self-reported), $n(\%)$ & $19(58 \%)$ \\
Independent & $7(21 \%)$ \\
Help with 1-2 ADLs* & $7(21 \%)$ \\
Help with 3+ ADLs & $23(70 \%)$ \\
Race/ethnicity, $n$ (\%) & $5(15 \%)$ \\
White & $3(9 \%)$ \\
Black & $2(6 \%)$ \\
Latino/Latina & $7(21 \%)$ \\
Asian/Pacific Islander & $7(21 \%)$ \\
High school diploma, GED ${ }^{\dagger}$, or less & $6(18 \%)$ \\
Marital status & $10(30 \%)$ \\
Single or never married & $10(30 \%)$ \\
Married or partnered & \\
Widowed & $30(91 \%)$ \\
Divorced & $3(9 \%)$ \\
Housing type & $9(27 \%)$ \\
Private residence & $12(36 \%)$ \\
Group home or supervised housing & $12(36 \%)$ \\
Clinic type & \\
General primary care & \\
Geriatrics primary care & Women's health
\end{tabular}

*Activities of daily living

tGeneral education diploma
Table 2 Themes Related to Measuring Functional Status in Primary Care

\begin{tabular}{ll}
\hline \hline Category & Themes \\
\hline $\begin{array}{l}\text { Importance of measuring and } \\
\text { discussing function in primary } \\
\text { care }\end{array}$ & $\begin{array}{l}\text { (1) Measuring function is part of } \\
\text { quality, holistic care } \\
\text { (2) Measuring function is not } \\
\text { medically relevant } \\
\text { (3) Providers are not equipped to } \\
\text { help with functional decline } \\
\text { (1) Interdisciplinary approaches } \\
\text { optimize measurement of function } \\
\text { (2) Face-to-face assessment is inti- } \\
\text { mate and accessible } \\
\text { (3) Self-assessment is less invasive } \\
\text { and less threatening } \\
\text { (1) Difficulty and need for help are } \\
\text { distinct yet interrelated concepts } \\
\text { (2) Providing context is essential } \\
\text { when discussing sensitive topics } \\
\text { such as functional decline }\end{array}$ \\
\hline
\end{tabular}

decline. A 74-year-old independent woman explained, "[discussing function is] important because [of] getting physical therapy or seeing what they can do to assist. If I don't say anything, she would not know if I'm having pain." Others explained that discussing function was important to address current and anticipated impairments. As an independent 75year-old woman said, "If [ADL impairment] were to happen, sure, I'd talk to her about it. I'd come running up here fast."

Measuring Function Is Not Medically Relevant. A minority of participants reported that discussing function was not important, typically because the participants did not have difficulty with ADLs or IADLs. As one 77-year-old man commented, "I don't have problems with these things. There's not much to talk to the doc about." Some of these participants, however, acknowledged that future difficulty with activities could influence their views. A 73-year-old man explained, "It's hard for me to think of this being difficult any time soon because I'm doing okay. So, I don't know... [all the ADLs and IADLs] are very important if I start having a problem."

Among some caregivers of functionally dependent patients, discussing function was less important because the patient either did not do a particular activity or already had the help they needed. As one caregiver of an 82-year-old man needing help with 1-2 ADLs explained, "none of these [questions] really relate to him" because he lives in a facility where he receives help with daily activities. Another woman who cared for her 88-year-old father explained that it was not important to discuss function with providers "because he's in a family setting and has help. I think if my dad lived in a different city and I went to see him and saw that the house was a mess...then I would bring it up to the doctor. But because he lives with me, that's not really an issue."

Another common theme among functionally independent patients was that IADLs were unrelated to medical care or outside the provider's scope of practice. Instead, they described IADLs as relevant only to their private social and domestic realms. For example, one 83-year-old independent 
man said, "[My doctor's] got enough to do taking care of the [medical problems]. He doesn't really have time and [there is] no benefit for him to get into my social activities, my house kind of activities." Others framed IADLs as "lifestyle" concerns not relevant to primary care. One 67-year-old independent man said, "I don't want to talk about any of this stuff. If you're asking about my heart, and my swelling, and my ankles, you know, my physical things, [that's okay]. You shouldn't be asking about my lifestyle." Similarly, a 77-yearold independent man viewed IADLs as external to medical practice, stating that "They [doctors] don't have the knowledge in that area, so they're stepping out of where they should be. I wouldn't ask a banker about my health either."

\section{Providers Are Not Equipped To Help with Functional} Impairment. A cross-cutting theme among all patient groups regardless of functional status was that providers are not equipped to help with functional impairment, in part because functional decline is an expected part of the aging process. One 67-year-old independent man explained, "Using the bathroom, I suppose [my provider] could do testing, and he could give me meds if I was having trouble going or something, but the rest of the stuff...most of it just looks like stuff that happens to you when you get older." A 68-year-old independent woman echoed the perception that providers cannot address functional impairment, stating "[My provider] couldn't help me with getting in and out of [bed]. She doesn't have the power to help me with my housework or anything."

We used these themes to develop Figure 1, which illustrates the relationship between patient functional status, perspectives, and themes related to the importance of discussing function in primary care.

\section{Preferences for Measuring Functional Status in Primary Care}

We identified several themes related to preferences for measuring function: (1) interdisciplinary approaches optimize measurement of function, (2) face-to-face assessment is intimate and accessible, and (3) self-assessment is less invasive (Table 2).

Interdisciplinary Approaches Optimize Measurement of Function. Participants were generally open to assessment from any primary care team member and viewed nurses and providers as playing complementary roles on an interdisciplinary team. A 76-year-old independent woman explained, "I think it's good to be asked questions by the nurse and the doctor, because maybe the nurse didn't relate to the doctor yet what she asked me. Maybe they just want to make sure I'm telling the same thing. I wouldn't have a problem with that." A caregiver of a 74-year-old functionally dependent man highlighted how an interdisciplinary approach to measuring function can improve care: "Share the knowledge as far as I'm concerned. The more they know about him, the better they can assess him and the better care they can give him."

Face-to-Face Assessment Is Intimate and Accessible. Another common theme related to patients' preference for measurement was that compared to self-assessment, face-toface assessment from a provider was more "intimate" and could prompt reflection on one's limitations, leading to more complete and accurate information. One 77-year-old independent man said, "I do prefer it, yeah, face-to-face. I mean, it's good for the prompting. I may be thinking about a [certain] problem, and actually I should be thinking about something else." Others commented that in-person assessment could prompt self-reflection among patients who did not perceive function to be relevant to medical care or who might be in denial about their level of impairment. A 73-year-old independent woman explained, "whenever there's a change in your abilities and you feel like you're diminished...you don't want to accept it. You know, you're sort of in denial...I think it's nice to have an outside person ask you, just because on their own you might not be willing to acknowledge certain things to yourself."

Among participants who preferred face-to-face assessment, several noted the loss of human connection when completing self-assessments, particularly through electronic data collection methods. As a 67-year-old independent man said, "The machine doesn't empathize with you. It doesn't know how you're feeling about the questions or the answers, you know? You can't be intimate with the machine like I can be with someone." Other responses illustrating participants' dislike of electronic self-assessments included low technology literacy (e.g., "I don't know how to use it") or a general aversion to technology (e.g., "I don't want to work with nobody's iPad"; "I'm not a computer person"). Other participants noted concerns over data security (e.g., "the net's notorious") and that selfreported information would not reach providers (e.g., "too many holes in the Swiss cheese"). Several participants also noted that physical impairments could make electronic or paper self-assessment challenging (e.g., "it's too difficult with my eyesight"; "I'm getting so my writing is not very good anymore").

Self-Assessment Is Less Invasive. In contrast, some participants preferred self-assessment because they perceived it as less invasive. This theme was common among participants who felt pressured or uncomfortable in interpersonal interactions and preferred a more "impersonal" experience. One 77-year-old independent man explained that although "it's not very personal putting it on a piece of paper, it allows me to think more about the question." A 71-year-old independent woman said, "I am more comfortable sending an email than actually talking to somebody. I have to really work at it when it comes to personal stuff." 


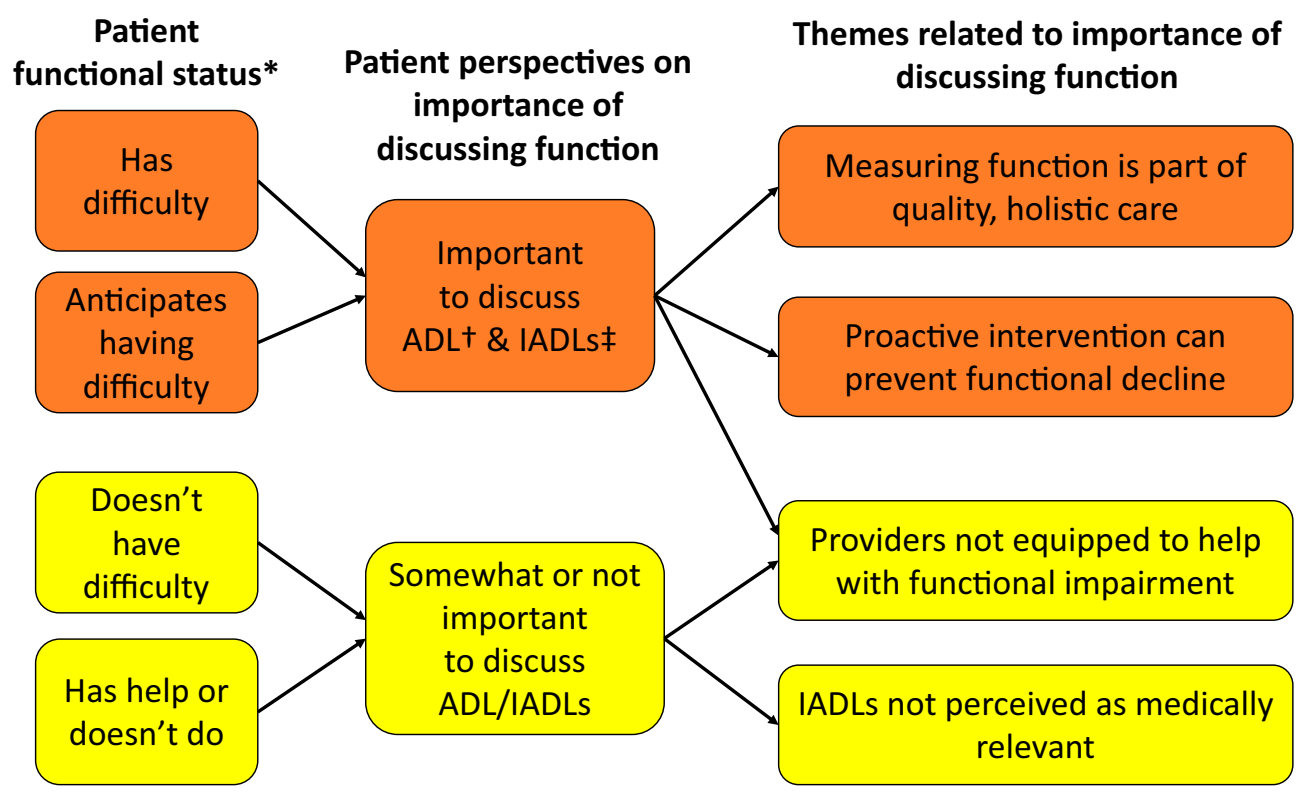

Figure 1 Relationship of patient functional status, perspectives, and themes related to the importance of discussing function in primary care. The model illustrates the relationship between patient functional status, perspectives on the importance of discussing function with providers, and the themes related to these perspectives. On the left, the model illustrates the functional status reported by patients, then links this status to possible perspectives in the middle of the figure: functional status is important, somewhat, or not important to discuss in primary care. The model then connects these perspectives to related themes which emerged from interviews, illustrated on the right side of the figure. These themes help to explain patient perspectives regarding the importance of discussing functional status. *Ability to perform ADL/IADLs, ${ }^{\dagger}$ activity of daily living, ${ }^{*}$ instrumental activity of daily living.

\section{Preferences for Communicating About Function}

Participants had varied preferences regarding communicating about function, illustrated by the following themes: (1) difficulty and need for help are distinct yet interrelated concepts and (2) providing context is essential when discussing sensitive topics such as functional decline.

\section{Difficulty and Need for Help Are Distinct Yet Interrelated} Concepts. Participants described how asking about difficulty performing daily tasks was a necessary precursor to asking about help. As an 81-year-old woman explained, "I don't have any difficulty, so why would I worry about if I need help right now?" Others noted conceptual differences between asking about difficulty and need for help, suggesting that both questions are important for providers to fully understand function. A caregiver of an 82-year-old man needing help with 1-2 ADLs stated, "I think they're two different questions. I would prefer that they were separate. Does he have trouble? Does he require help?" Participants' interpretation of the meaning of the assessment questions influenced their preference for question wording. For example, several participants perceived that being asked if they needed help with daily tasks implied weakness or lack of independence. As a 77-year-old man noted, "I'm an independent guy and I don't like help." In contrast, a few participants indicated that being asked about difficulty was "offensive" and that asking about help was "probably a little softer and nicer than saying, 'Are you having difficulty?" A 71-year-old independent woman explained, “'Do you need help?' sounds less scary to me than, 'Is it difficult?' When I hear, 'Is it difficult,' my little brain is going like, 'uh-oh, they're evaluating me. They're going to lock me up [in a nursing home] or something."”

\section{Providing Context Is Essential When Discussing Sensitive}

Topics. Participants also emphasized the importance of providing context when asking questions about function, a potentially sensitive topic. As a 74-year-old independent woman noted, when a provider does not explain why they are asking about function, "it's too pointed...It's not what you say, it's how you say it. [Asking about difficulty rather than help] sounds more like you're caring." She also emphasized the importance of rapport and communication style, suggesting that providers preface questions about function by asking, “'How are you feeling today?' To me, you open a person up that way." Patients also emphasized the importance of using functional status data to inform care, such as making referrals for services and supports. As one 81-year-old functionally dependent woman explained, "The questions don't bother me, as long as there's a result to come out of it."

\section{DISCUSSION}

In this qualitative study, older veterans and caregivers reported varying perspectives on measuring function in primary care. Overall, participants felt that measuring and discussing function was important to allow primary care teams to provide optimal care. However, a less common theme was that 
assessing function was not relevant to them or was not a "medical" issue. Most participants preferred face-to-face over self-assessment and noted the importance of assessing both difficulty and need for help with daily activities. Participants also emphasized the importance of providing context for questions and using information about function to inform care. These findings suggest that incorporating patient preferences for measuring function has the potential to improve patient satisfaction and experience with functional assessment in primary care.

Patients emphasized the importance of assessing function to provide optimal care, including maintaining quality of life, preventing functional decline and addressing current impairments. This finding is consistent with research that shows the importance of assessing patient-reported outcomes to address the issues that are most important to patients and integral to quality of life. ${ }^{4,5,31}$ A subset of patients, however, did not think that discussing function was important, often because they viewed tasks such as managing finances as outside the medical realm. This perception points to some of the unique challenges of assessing function in primary care. Unlike chronic conditions such as diabetes or hypertension, ADLs and IADLs may be perceived as personal, intimate topics that a provider has no business addressing or is unable to alleviate. Yet IADL impairment is one of the first signs of cognitive impairment, ${ }^{32}$ and functional impairment strongly predicts adverse health outcomes. ${ }^{1-3}$

This gap between patients' perception of function's relevance to health and its actual prognostic importance emphasizes the need for primary care teams to provide context when assessing function. Educating patients and families about the relevance of function to medical care may help alleviate concerns that function is solely relevant to nursing home admission. Patients and families may also benefit from education about the role that primary care providers play as gatekeepers in referring patients with impairment to appropriate multidisciplinary professionals. $^{33,} 34$ Social workers, physical therapists, and occupational therapists play a critical role in helping older adults to improve and maintain function. Greater coordination and integration between primary care and these providers may help ensure patient access to services that can improve function. ${ }^{35,36}$

Preferences for measuring function in primary care were remarkably consistent in our study. Patients reported being open to assessment from any primary care team member and valued an interdisciplinary approach to assessment. In busy primary care settings, interdisciplinary approaches that include screening by a nurse followed by assessment by a provider may help ensure routine, standardized measurement of function; this strategy is already being successfully used in some clinics and has been identified as a facilitator to routine measurement of function. ${ }^{18,37}$ Further, previous work has shown that clinicians are cautious about additional workload burdens that do not relate directly to patient care. By enhancing clinician knowledge of patient perspectives regarding functional status, clinicians may be able to more effectively communicate the importance and relevance of measuring function to address current or future impairments.

Our findings further show that most older adults preferred face-to-face functional assessment over self-assessment. Selfassessment of symptoms and quality of life among adults has been shown to be acceptable in some clinic settings. ${ }^{38,} 39$ However, our findings suggest that for many older adults, functional assessment may be most acceptable when it is performed face-to-face, due to patients' needs for context, empathy, and understanding of function's relevance to health care. Additionally, some older adults expressed a reluctance to engage in electronic self-assessment due to concerns about ease of use, lack of human connection, and data security. Our findings are consistent with research showing that older adults may be wary about engaging with technology if they anticipate a steep learning curve ${ }^{40}$ or perceive technology to be a substitute for human interaction. ${ }^{41,42}$ While electronic selfassessments hold promise for streamlining assessments in primary care, ${ }^{43}$ our findings suggest that older adults' concerns about electronic assessment must be considered when developing approaches to measure function.

Participants had specific preferences for question wording, distinguishing between asking about difficulty with daily activities and the need for help. This finding aligns with research showing that these are distinct, complementary concepts that contribute to a holistic understanding of a patient's function. ${ }^{44}$ Further, some participants expressed concern that asking about need for help without first asking about difficulty can seem abrupt or may reflect the providers' intention to place them in a nursing home. This finding is consistent with research showing that some older adults feel stigmatized when asked about needing help with daily activities. ${ }^{45}$ Participants also emphasized the importance of providing context when asking about function, noting that without explanation, these questions can seem invasive.

Overall, these findings highlight the unique aspects and challenges of functional status measurement, including its relevance across medical and social realms and the importance of contextualizing discussions of function. To address these challenges, approaches to accurately and sensitively assess function in older adults must include education for primary care teams in how to contextualize functional measurement, assessments of both difficulty and need for help, and systems to efficiently link patients with appropriate referrals.

This study has several limitations. Patients may have been more likely to enroll if they viewed discussing daily activities in primary care as important. We took several measures to ensure a diverse sample, including sending letters to all eligible patients and stratifying recruitment by race, gender, and function. Most of our sample was White and had greater than a high school education. Because of the 
demographics of older veterans and the benefits of the G.I. Bill, ${ }^{46}$ this is reflective of the older veteran population. Although our sample did include nearly one-third racial/ethnic minorities, our findings may not be generalizable to more diverse populations.

In conclusion, older veterans expressed the importance of measuring function in primary care, preferred face-to-face assessments, and emphasized the importance of providing context when asking questions about function. These findings suggest that to more effectively assess function, we must consider patient preferences. Approaching function from a patient-centered perspective, including sensitivity to method of assessment and communication style, has the potential to improve patient satisfaction and willingness to provide functional status information in primary care.

Corresponding Author: Francesca M. Nicosia, PhD; Division of Geriatics, Department of Medicine of the University of California, San Francisco, San Francisco, CA, USA (e-mail: Francesca. nicosia@ucsf.edu).

Author Contributions The authors do not have any additional contributors to report.

Funding Information This work was supported by grant QUE 15283 (Brown) from the Veterans Affairs (VA) Quality Enhancement Research Initiative in partnership with the VA Office of Geriatrics and Extended Care. This work was additionally supported by grants from the National Institute on Aging at the National Institutes of Health (grant numbers K23AG045290 to Brown, K76AG057016 to Brown, P3OAG044281 to Brown); the National Institutes of Health National Center for Advancing Translational Sciences through the University of California, San Francisco, Clinical and Translational Science Institute (grant number KL2TROOO143 to Brown); and the American Federation for Aging Research (grant number K76AG057016 to Brown). These funding sources had no role in the preparation, review, or approval of the manuscript. Dr. Brown is an employee of the Veterans Health Administration. The opinions expressed in this manuscript may not represent those of the Department of Veterans Affairs.

\section{Compliance with Ethical Standards:}

Conflict of Interest: The authors do not have any conflicts of interest, financial or personal, to report and have emailed completed disclosure forms to the Assistant Managing Editor.

\section{REFERENCES}

1. Gaugler JE, Duval S, Anderson KA, Kane RL. Predicting nursing home admission in the U.S: a meta-analysis. BMC Geriatr. 2007;7:13.

2. Inouye SK, Peduzzi PN, Robison JT, Hughes JS, Horwitz RI, Concato J. Importance of functional measures in predicting mortality among older hospitalized patients. JAMA. 1998;279(15):1187-1193.

3. Carey EC, Walter LC, Lindquist $\mathbf{K}$, Covinsky KE. Development and validation of a functional morbidity index to predict mortality in community-dwelling elders. J Gen Intern Med. 2004;19(10):1027-1033.

4. Fried TR, McGraw S, Agostini JV, Tinetti ME. Views of older persons with multiple morbidities on competing outcomes and clinical decision making. J Am Geriatr Soc. 2008;56:1839-44

5. Fried TR, Bradley EH, Towle VR, Allore H. Understanding the treatment preferences of seriously ill patients. N Engl J Med. 2002;346: 1061-6.
6. Fried TR, Tinetti ME, Iannone L, O'Leary JR, Towle V, Van Ness PH Health outcome prioritization as a tool for decision making among older persons with multiple chronic conditions. Arch Intern Med. 2011;171:1854-6.

7. Cenzer IS, Tang V, Boscardin WJ, et al. One-year mortality after hip fracture: development and validation of a prognostic index. J Am Geriatr Soc. 2016;64:1863-8.

8. Kurella Tamura M, Covinsky KE, Chertow GM, Yaffe K, Landefeld CS, McCulloch CE. Functional status of elderly adults before and after initiation of dialysis. N Engl J Med. 2009;361:1539-47.

9. Hurria A, Togawa K, Mohile SG, et al. Predicting chemotherapy toxicity in older adults with cancer: a prospective multicenter study. J Clin Oncol. 2011;29:3457-65

10. Crawford RS, Cambria RP, Abularrage CJ, et al. Preoperative functional status predicts perioperative outcomes after infrainguinal bypass surgery. J Vasc Surg. 2010;51:351-8.

11. Arnold SV, Reynolds MR, Lei Y, et al. Predictors of poor outcomes after transcatheter aortic valve replacement: results from the PARTNER (Placement of Aortic Transcatheter Valve) trial. Circulation. 2014;129:2682-90.

12. Walter LC, Covinsky KE. Cancer screening in elderly patients: a framework for individualized decision making. JAMA. 2001;285:2750-6.

13. Bogardus ST Jr, Towle V, Williams CS, Desai MM, Inouye SK. What does the medical record reveal about functional status? A comparison of medical record and interview data. J Gen Intern Med. 2001;16:728-36.

14. Bierman AS. Functional status: the six vital sign. J Gen Intern Med 2001;16:785-6.

15. Calkins DR, Rubenstein LV, Cleary PD, et al. Failure of physicians to recognize functional disability in ambulatory patients. Ann Intern Med. 1991;114:451-4.

16. Schor EL, Lerner DJ, Malspeis S. Physicians' assessment of functional health status and well-being. The patient's perspective. Arch Intern Med. 1995; 155:309-14

17. Iezzoni LI, Greenberg MS. Capturing and classifying functional status information in administrative databases. Health Care Financ Rev. 2003;24:61-76.

18. Nicosia FM, Spar MJ, Steinman MA, Lee SJ, Brown RT. Making function part of the conversation: clinician perspectives on measuring functional status in primary care. J Am Geriatr Soc. 2019;67:493-502.

19. Brown RT, Komaiko KD, Shi Y, et al. Bringing functional status into a big data world: validation of national Veterans Affairs functional status data. PLoS One. 2017;12:e0178726.

20. Costley AW. A turning point in functional assessment: from a medical view to a social view of difficulty with daily activities. Int J Aging Soc. 2013;2:83-91

21. Palinkas LA, Horwitz SM, Green CA, Wisdom JP, Duan N, Hoagwood K. Purposeful sampling for qualitative data collection and analysis in mixed method implementation research. Adm Policy Ment Health. 2015;42:533-44.

22. Sudore RL, Landefeld CS, Williams BA, Barnes DE, Lindquist $\mathbf{K}$, Schillinger D. Use of a modified informed consent process among vulnerable patients: a descriptive study. J Gen Intern Med. 2006;21:867-73.

23. Albert SM, Bear-Lehman J, Burkhardt A. Lifestyle-adjusted function: variation beyond BADL and IADL competencies. Gerontologist. 2009;49:767-77.

24. Baum CM, Edwards DF. The Washington University Activity Card Sort St. Louis, MO: Penultimate Publications; 2001.

25. Given LM. The SAGE Encyclopedia of Qualitative Research Methods. Thousand Oaks, CA: SAGE; 2008.

26. Charmaz K. Constructing Grounded Theory: a Practical Guide Through Qualitative Analysis. 2 ed. Thousand Oaks, CA: SAGE; 2006.

27. Foley G, Timonen V. Using grounded theory method to capture and analyze health care experiences. Health Serv Res. 2015;50:1195-1210.

28. Bowen G. Naturalistic inquiry and the saturation concept: a research note. Qual Res. 2008; 8(1):137-142.

29. O'Reilly M, Parker N. 'Unsatisfactory saturation': a critical exploration of the notion of saturated sample sizes in qualitative research. Qual Res. 2013; 13(2):190-197.

30. Guidance Regarding Methods for De-identification of Protected Health Information in Accordance with the Health Insurance Portability and Accountability Act (HIPAA) Privacy Rule [Internet]. U.S. Department of Health and Human Services. 2015 [cited 2019 Nov 10]; Available from: https://www.hhs.gov/hipaa/for-professionals/privacy/special-topics/ de-identification/index.html\#_edn 1 . 
31. Nelson EC, Hvitfeldt Forsberg H, Reid R, et al. Using patient-reported information to improve health outcomes and health care value: case studies from Dartmouth, Karolinska and Group Health. Dartmouth Institute for Health Policy and Clinical Practice. 2012.

32. Widera E, Steenpass V, Marson D, Sudore R. Finances in the older patient with cognitive impairment: "He didn't want me to take over". JAMA. 2011;305:698-706.

33. Forrest CB. Primary care in the United States: primary care gatekeeping and referrals: effective filter or failed experiment? BMJ. 2003;326:692-5.

34. Ploeg J, Denton M, Hutchison B, et al. Primary care physicians perspectives on facilitating older patients' access to community support services: qualitative case study. Can Fam Physician. 2017;63:e31-e42.

35. Mann CC, Golden JH, Cronk NJ, Gale JK, Hogan T, Washington KT Social workers as behavioral health consultants in the primary care clinic. Health Soc Work. 2016;41:196-200.

36. Donnelly C, Brenchley C, Crawford C, Letts L. The integration of occupational therapy into primary care: a multiple case study design. BMC Fam Pract. 2013;14:60.

37. Spar MJ, Nicosia FM, Steinman MA, Brown RT. Current approaches to measuring functional status among older adults in VA primary care clinics. Fed Pract. 2017;34:26-31.

38. Maguire R, Ream E, Richardson A, et al. Development of a novel remote patient monitoring system: the advanced symptom management system for radiotherapy to improve the symptom experience of patients with lung cancer receiving radiotherapy. Cancer Nurs. 2015;38:E37-47.
39. Wolpin S, Berry D, Austin-Seymour M, et al. Acceptability of an electronic self-report assessment program for patients with cancer. Comput Inform Nurs. 2008;26:332-8.

40. Brahmandam S, Holland WC, Mangipudi SA, et al. Willingness and ability of older adults in the emergency department to provide clinical information using a tablet computer. J Am Geriatr Soc. 2016;64:2362-7.

41. Currie M, Philip LJ, Roberts A. Attitudes towards the use and acceptance of eHealth technologies: a case study of older adults living with chronic pain and implications for rural healthcare. BMC Health Serv Res. 2015;15: 162

42. Heinz M, Martin P, Margrett JA, et al. Perceptions of technology among older adults. J Gerontol Nurs. 2013;39:42-51.

43. Powell RE, Henstenburg JM, Cooper G, Hollander JE, Rising KL. Patient perceptions of telehealth primary care video visits. Ann Fam Med. 2017; 15:225-9.

44. Gill TM, Robison JT, Tinetti ME. Difficulty and dependence: two components of the disability continuum among community-living older persons. Ann Intern Med. 1998;128:96-101.

45. Costley A. Rethinking aging and daily "difficulties": from functional bodies to functional communities. Anth Aging Quart. 2012;33:157-163

46. Bound $\mathbf{J}$, Turner $\mathbf{S}$. Going to war and going to college: did World War II and the G.I. Bill increase educational attainment for returning veterans? J Labor Econ. 2002;20:784-815

Publisher's Note Springer Nature remains neutral with regard to jurisdictional claims in published maps and institutional affiliations. 